\title{
In Response: Safe Triangle Technique
}

We appreciate the comments in this editorial regarding the need to avoid the "safe triangle technique" or as we and others like to call it the "unsafe triangle technique". We agree with your comments that placing the tip of the needle as far way from the radicular artery is important to further reduce the risk of injury to the radicular artery and any subsequent neurological deficits.

We agree with your comments about placing the needle tip in the inferior aspect of the foramen. We are in the process of finishing a prospective observational study that looks at transforaminal injections with placement of the needle tip in the posterior aspect of the inferior portion of the foramen. This approach has its advantages and disadvantages as we will comment in this paper. The big advantage of course is being as far away from the radicular artery as possible. In addition, the posterior positioning helps to avoid the inferiorly located foraminal vein(s). The disadvantage of this approach is in patients with stenosis of the inferior aspect of the foramen from a disc protrusion, posterior vertebral osteophyte or facet joint hypertrophy which makes it technically difficult or impossible to place the needle tip in the inferior aspect of the foramen.

The three mechanisms by which foraminal stenosis occurs are SAP hypertrophy (foraminal stenosis), lateral recess stenosis (ex. vertebral spondylosis or osteophyte) and disk protrusion (Figure 1). All three of these structures are located in, and cause stenosis of the inferior aspect of the foramen. Thus, targeting the inferior aspect of the foramen may increase the difficulty of placing the needle tip into the posterior epidural space to achieve the dye pattern described in the article (1).

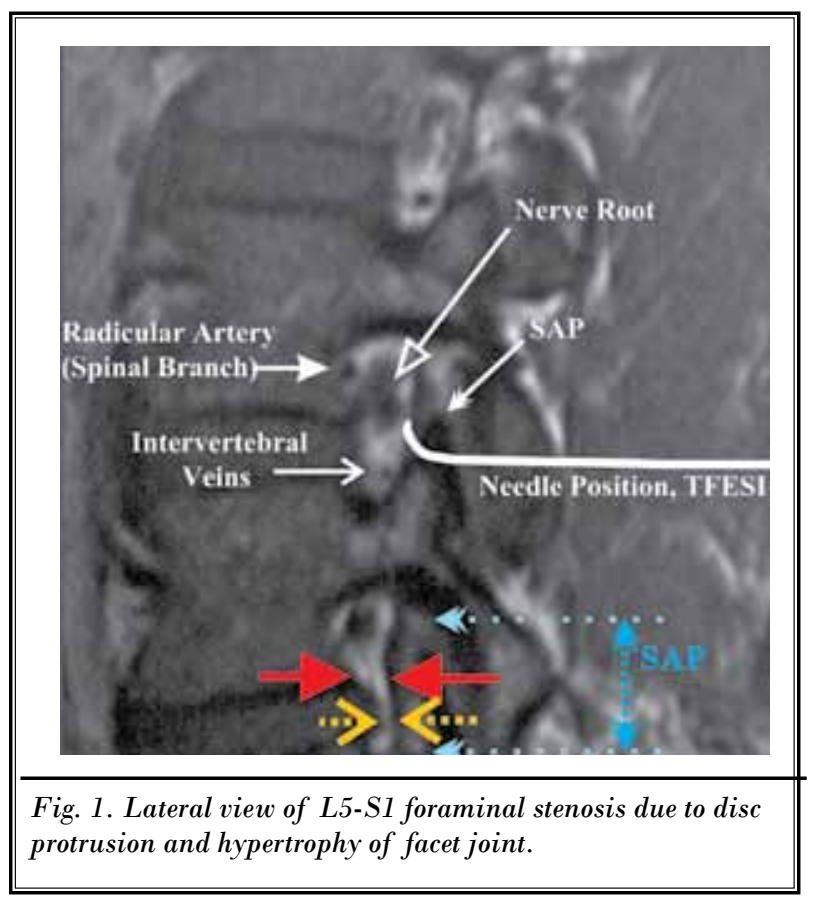

(The goal of this approach is to inject steroid into the epidural space around the nerve root without compromising vascular supply to the spinal cord). Additionally, caution should be taken with this modification because it may increase the risk of injection of contrast into the outer layer of the protruded disc due to its proximity to posterior wall of the SAP.

Thus the more rostral posterior approach is the best option in these circumstances. Obviously, if the foraminal stenosis is severe with narrowing of the entire 
foramen than even the posterior approach can be difficult or impossible to perform safely.

Another disadvantage of the inferior approach is that you loose your ability to inject specific nerve root levels. The contrast flow with this technique typically spreads across the epidural space as oppose to along the nerve root (at low volumes) which essential produces an epidural (transforaminal epidural) injection that will have an effect on the passing nerve roots and not the exiting nerve root. Contrast flow eventually will flow along the exiting nerve root at larger volumes, but in dong so there is a greater volume of injectant in to the epidural space including the spread to the contralateral sides in some cases.

So, the inferior or inferior posterior as well as the posterior neural approach has its limitations. Never the less, the advantages far out way the disadvantages if it indeed reduces or eliminates the risk of a radicular artery injury and the neurological deficits associated with this procedure.

Jie Zhu, MD

Attending Physician

Mid Atlantic Spine \& Pain Physicians

Newark, DE

Adjunct Assistant Professor

Temple University Medical School

Philadelphia, PA.

Email: zzzzjim@gmail.com

Frank J.E. Falco, MD

Medical Director

Mid Atlantic Spine \& Pain Physicians

139 E. Chestnut Hill Rd.
Newark, DE

Director

Pain Medicine Fellowship Program

Temple University Hospital

Philadelphia, PA

Adjunct Associate Professor

Temple University Medical School

Philadelphia, PA.

Ferdinand Formoso, DO

Attending Physician

Coastal Pain and Spine Center

Jacksonville, FL

Obi Onyewu, MD

Attending Physician

Mid Atlantic Spine \& Pain Physicians

Newark, DE

Faculty

Pain Medicine Fellowship Program

Temple University Hospital

Philadelphia, PA

Adjunct Assistant Professor

Temple University Medical School

Philadelphia, PA

Franklin L. Irwin, MD

Attending Physician

Mission Pain and Spine

Mission Viejo, CA

\section{References}

1. Devereaux MW, Anatomy and examination of the spine. Neurol Clin 2007; 25:331-351.

2. Zhu J, Falco FJE, Formoso F, Onyewu O, Irwin FL. Alternative approach for lumbar transforaminal epidural steroid injections. Pain Physician 2011; 14:331-341. 